Probiotics and Microbiome

\title{
The Impact of Microbiome on Childhood Asthma
}

\begin{abstract}
Aida Semic-Jusufagic
Department of Paediatric Allergy, Leeds Children's Hospital, Great George Street, Leeds, LS1 3EX Leeds Teaching Hospitals NHS Trust and University of Leeds, United Kingdom

Correspondence: aida.semic@nhs.net

Tel.: + 441133922441

Fax.: + 441133928617

Received: December 3, 2018

Accepted: January 12, 2019

Key Words: Microbiome - Microbiota • Childhood Asthma • Gut-Lung Axis.

This article is a review of current evidence on influence of microbiome on asthma development. The prevalence of asthma and allergic disease has been on constant increase during the last century. The scientific community has begun to understand more about possible factors that have contributed to the epidemic. Such sharp rise in prevalence cannot be explained by genetic factors as these changes occur over much longer period of time. Stemming from research based on environmental factors and 'hygiene hypothesis', there is a large body of evidence that commensal microbiota is an important regulator of early life immune system development and could protect against subsequent asthma and allergic disease. This review is focused on a broader and mechanistic context of hygiene hypothesis reviewing evidence on how gut and respiratory microbiota shape immune responses and influence asthma risk during childhood. Microbial metabolites, diet and its interaction with host immune system are discussed. Also, dynamics of respiratory microbiota, its determinants and interactions with viral infections during early life that predispose to subsequent childhood asthma are summarised. Conclusion - The composition of gut and respiratory microbiome could determine the risk of childhood asthma development.
\end{abstract}

\section{Introduction}

There is a growing body of evidence on importance of microbiome in determining the status between health and disease. A number of diseases such as atopic diseases and autoimmune disorders have been linked to perturbation in commensal microbial communities.

The aim of this article is to review current evidence on association between microbiome and asthma in childhood.

\section{Asthma and Hygiene Hypothesis}

The increase in asthma and allergic disease has paralleled major lifestyle changes that could have influenced different aspects of im- munological and physiological development during the childhood. Several epidemiological factors have been widely debated as possible contributors to such rise in developed countries, for example: 1) improvements in public hygiene resulting from supply of clean water have resulted in eradication of enteric diseases, 2) changes in agricultural methods contributing to intense farming and higher pollen counts, 3) better living conditions have contributed to sedentary lifestyle with children a) spending longer time indoors being for longer exposed to indoor allergens, b) reduced physical activity and decreased fitness c) introduction of children's TV programme and resulting change in breathing 
pattern and d) rise in obesity compared to previous generations (1).

Following inception of hygiene hypothesis in late 1980's which observed association between hay fever and small family size (2), it has been postulated that early life microbial exposures in infancy provide necessary stimulation for the immune system development and prevention of $\mathrm{T}_{\mathrm{H}} 2$ immune milieu seen in asthma and atopy. Whether all microbial exposures are protective of asthma is matter of controversy as certain viral infections such as rhinoviruses and respiratory syncytial virus (RSV) have been shown to be risk factors for the development of asthma (3). Whilst allergic sensitisation is known to increase the risk of childhood asthma by several folds (4), the timing of the allergen exposure in relation to infection and its severity may play important role. In murine model concurrent moderate intensity infection with $C$. pneumoniae and allergen exposure predisposed to eosinophilic airway inflammation, whereas severe infection or later exposure to allergen did not cause same effect (5).

Growing up in households with the dog is protective against development of allergic disease (6). The effect is mediated through exposure to bacteria-enriched house dust that can stimulate immune system development and prevent allergen- mediated airway response (7). Several ground-breaking epidemiological studies have demonstrated that exposure to non-pathogenic microorganisms through dust or unpasteurised milk in children living in farming environments is protective against development of childhood asthma (3). In study by Ege et al. asthma and atopy were less prevalent in children living in traditional farming environment compared to those living in urban setting (8). It has been proposed that the protection against asthma could be mediated through exposure to diverse microbial organisms. These microbial exposures occur through exposure to farm dust, direct contact with farm animals and drinking of unpasteurised milk (9).

An excellent comparison on how farming may influence development of asthma comes from the US, where two distinctive farming communities of Amish and Hutterite have been shown to have a four-fold difference in prevalence of asthma and allergic sensitisation $(5.2 \%$ vs $21.3 \%$ and $7.2 \%$ vs.33.3\%, respectively) (9). Both communities share European genetic ancestry and lead similar lifestyle with the main disparity being in traditional or industrialised farming, respectively. The striking difference in prevalence of asthma and allergic disease between two communities can be explained by much higher exposure to endotoxin in house dust of Amish in comparison to Hutterite homes. Amish children also had significantly lower levels of total and specific immunoglobulin E (IgE) production, lower eosinophils and higher proportion of neutrophils in peripheral blood. The same endotoxin has been shown to stimulate innate immunity via regulation of tumor necrosis factor (TNF) and interferon regulatory factor 7 (IRF7) expression in experimental model of asthma. Although study did not examine components of endotoxin specifically, the findings suggest that the house dust samples of two communities have unique microbial compositions.

Alteration of gut microbiota composition through early life use of antibiotics for treatment of infections may predispose to the development of asthma and allergic disease. Delayed colonisation and alteration in earlylife gut microbiota can be caused by perinatal and postnatal antibiotic use. Studies have shown that full term infants who have received antibiotics in perinatal period have reduced bacterial gut diversity, less Lactobacilli and Bifidobacteria and higher percentage of Proteobacteria and Enterobacteria (10).

Many studies have shown positive relationship between early life antibiotic use and 
risk of childhood wheezing and asthma (11, 12), however no effect on atopy, suggesting that the association between asthma and antibiotics is not mediated through alteration of gut microbiota. Majority of antibiotic courses prescribed during first few years of life are prescribed for treatment of respiratory infection, which could be confounding factor when assessing the relationship between two. There could be other explanations to observed link such as susceptibility to severe respiratory viral infections in early life that could be a predisposing factor to both childhood wheezing and antibiotic prescribing. In Manchester birth cohort study (MAAS) we found that antibiotic use in the first year of life was associated with subsequent wheeze and asthma but also, poorer antiviral immune response to common respiratory viruses but not bacteria at age 11 years (13). Interestingly, antibiotic prescribing in first year of life was associated with variants in $17 \mathrm{q} 21$ region that have been previously found to increase the risk of childhood asthma, suggesting that same factor operating within context of increased susceptibility to viral infections might predispose to antibiotic use in infancy and asthma. Both COAST and COPSAC cohort studies have also confirmed association between $17 \mathrm{q} 21$ variants, rhinovirus induced wheezing and subsequent childhood asthma (3).

Overall, multiple factors associated with modern living have been associated with asthma and allergic disease development. It seems that dramatic changes in exposure to early life microbial organisms has left significant gap in the process of immune system regulation during the critical time period in lifetime. Some of these associates, such as use of antibiotics, may be merely a manifestation of other underlying risk factors for asthma development. Rather than inferring simplified version of hygiene hypothesis whereby sanitation, cleanliness and lack of common childhood infections are to blame for allergy epidemic, Rook et al. (14) have proposed that reduced microbial biodiversity/ exposure to commensals in early life and thereby lack of essential input into maturing immune system is a predisposing factor to overreactive immune response seen in allergies. Indeed, cleaning habits do not have lasting effect on microbial levels indoors as the microbial levels are constantly replaced. The authors argue that content of indoor dust has changed in urban homes as people moved away from rural surroundings resulting in overall reduced human skin and gut microbial diversity.

\section{Asthma and Gastrointestinal Microbiome}

Human kind has evolved by living together in symbiosis with large number of different commensal microorganisms residing at different anatomical body locations. Living in developed countries has contributed to microbial imbalance or dysbiosis which is described as a 'deviation from an optimal, health promoting microbial community through loss of beneficial microorganisms, expansion of potentially pathogenic microorganisms and loss of overall microbial diversity' $(14,15)$. The term microbiome has been defined as 'the sum of microbes, their genomic elements and interactions in a given ecological niche', whilst microbiota is defined as 'the sum of microbes found in given ecological niche' (15).

The composition of gut microbiota has been shown to influence immune regulation. Microbes and their products directly stimulate skewing of adaptive immune system from $\mathrm{T}_{\mathrm{H}} 2$ to $\mathrm{T}_{\mathrm{H}} 1 / \mathrm{T}_{\mathrm{H}} 17$ and $\mathrm{T}$ regulatory $\left(\mathrm{T}_{\text {reg }}\right)$ cells via gut- associated lymphoid tissue (GALT) or indirectly via gut-lung axis as demonstrated in murine models (14). The delay in gut microbial colonisation in germfree mouse model resulted in failure to develop GALT and skewing towards $\mathrm{T}_{\mathrm{H}} 2 \mathrm{im}$ mune responses (16). Longitudinal study has 
shown that composition of gut microbiota is already different in infants who go on to develop asthma even before any symptoms occur (17). This study looking at gut microbiota of 319 children in the first year of life found that the differences in abundance of certain bacterial species as early as 3 months of age predicted development of wheeze and atopy at age 12 months (18). In those with wheeze and atopy the depletion of Faecalibacterium, Lachnospira, Rothia and Veilonella was more likely to result in lower lypopolyssaccharide (LPS) and short-chain fatty acids (SCFA) acetate levels when compared to controls. Within the same study, a proof-ofconcept experimental inoculation of germfree, airway inflammation mouse model with bacterial species found to be depleted in children who had wheeze and atopy resulted in symptom improvement.

The effects of gut microbiome are not only localised to the gut as several experimental studies have demonstrated existence of gut-lung axis, whereby exposure to microbial components or SCFA also can provide necessary immune regulation and stimulate immune tolerance by reducing and protecting against allergic airway responses and inflammation (14). In animal model, heat-treated Enterococcus faecalis lysate administered orally has been shown to reduce $\mathrm{T}_{\mathrm{H}} 17$ cell immune response and prevent allergic airway inflammation (19). A recently discovered, low-presence gut bacteria, segmented filamentous bacteria (SFB) or Candidatus Savagella has been implicated in development of GALT, regulation of $\mathrm{T}_{\mathrm{H}} 17$ pathway genes and higher intestinal IgA secretion in first 3 years of life (20). These findings indicate that SFB may play significant role in postnatal maturation of immune response. Intestinal fluid of SFB - positive samples was associated with distinctive microbiota compared to SFB -negative samples, which were abundant in potentially pathogenic bacteria such as Bacteroides, Escherichia and Klebsiella, probably due to lower secretion of luminal IgA. On the other side, intestinal overgrowth of SFB in mice has been implicated in autoimmunity. SFB was found to trigger autoimmune lung disease in animal model of rheumatoid arthritis but not in controls, by increasing lymphocytic lung infiltration and expansion of lung mucosal $\mathrm{T}_{\mathrm{H}} 17$ cells that recognise both bacteria and self-antigen (21).

One of the ways that gut microorganisms have positive effect on immune regulation and prevention of asthma and allergic disease is through secretion of SCFA such as acetate, butyrate and propionate following fermentation of dietary fibres in colon (22). Although SCFAs can be directly sourced through diet, it is mostly derived by colonic bacteria. Not only SCFA are important source of energy for colonic cells, they also regulate organisation of tight junctions, promote dendritic cell regulatory activity and induce $\mathrm{T}_{\text {reg }}$ and IL-10 secreting $T$ cells. These effects are mediated through SCFAs ability to activate G-protein coupled receptors (GPCRs) that regulate transcription factors and prevent inflammation (23).

Other microbial derived mediators can have opposite effect and may boost allergic phenotype. Certain gut located bacterial species such as Escherichia coli, Morganella morganii and Lactobacillus vaginalis have been shown to secrete high levels of histamine and were found to be over-represented in the faecal samples of asthmatics compared to healthy controls (24). Interestingly, the highest presence of bacterial histamine decarboxylase enzyme (HDC) responsible for conversion of L-histidine to histamine was detected in non-obese asthmatics compared to obese asthmatics. This difference could not be explained by variance in their dietary habits, age, gender, obesity or difference in atopic status between two groups. Those with the highest faecal HDC levels were found to have the poorest lung function $\left(\mathrm{FEV}_{1}\right)$ among the asthma group. Of three bacterial species 
identified, non-obese asthmatics had highest levels of $M$. morganii detected compared to controls and obese asthmatics. This suggests that distinctive etiological factors determine these two asthma endotypes.

Breastfeeding could be important determinant of 'healthy' oral and gut microbiome. It is also known children who have been breastfed have better lung function and it also may protect against asthma development (17). Breast milk is unique as it contains many bioactive nutrients including human milk oligosaccharides (HMO) and microbiota. HMOs act as prebiotics in lower gut acting as a substrate for gut microbiota but have been also found to reach lungs via systemic circulation. It has been postulated that prebiotics could be directly interacting with lung epithelia, immune cells and lung microbiota preventing asthma development (17). Breast milk is a rich source of Bifidobacteria which can be depleted in favour of potential pathogens in infants who have had expressed breast milk. Interestingly, feeding with expressed breast milk was associated with increased risk of childhood asthma (25), suggesting that microbial composition and abundance of Bifidobacteria in breast milk play important role in immune system regulation. As well as breastfeeding, diet rich in anti-inflammatory nutrients such as unsaturated fatty acids can modify systemic inflammation seen in asthma (26). Westernised diet is poor in anti-inflammatory nutrients and fiber. Dietary fiber is important for viability of gut microbiota and its ability to produce SCFAs. In experimental models, high fiber intake supported Bacteroidetes and Actinobacteria and was associated with protection against airway inflammation.

In summary, gut microbiota composition is an important factor influencing immune system maturation. The imbalance in composition of gut microbiota in early life can be detrimental factor predisposing to development of asthma and atopic sensitisation.
Multiple environmental and lifestyle factors including diet and mode of breastfeeding have been individually identified to determine the risk. However, scientific and medical community are yet to define intervention strategies that could support ultimate microbiota composition that will support long term health and prevention of asthma and allergic disease development.

\section{Asthma and Respiratory Microbiome}

The link between respiratory infections and asthma has been long recognised and both viral and bacterial pathogens have been implicated. Human rhinovirus (HRV) and RSV- induced wheezing illness in infancy is a recognised risk factor for development of childhood asthma. There is emerging evidence that microbiota colonising mucosal surfaces can determine the state of health and the disease with more evidence regarding microbial dysbiosis in asthma $(27,28)$.

Lower airways are not sterile and have similar microbial composition to upper airways, therefore assessment of nasal microbiota can provide insight into asthma pathogenesis. Nasal bacterial colonisation with $S$. pneumoniae, $M$. cattarhalis and $H$. influenzae as early as first month of life and even before development of any respiratory infections, has been linked to diagnosis of asthma 5 years later (29). An Australian birth cohort study investigating the airway microbiome during the first year of life assessed changes in nasopharyngeal (NP) microbiome at health and during respiratory illness and its association with subsequent asthma (30). Healthy infants' NP was predominately colonised with Staphylococcus or Corynebacterium at 2 months of age, with shift towards predominantly Alloiococcus (or Dolosigranulum) and Moraxella at one year of age. During the acute respiratory illness (ARI) NP samples showed abundance in Streptococcus, Morax- 
ella and Haemophilus genera. The association remained unchanged even after adjusting for concurrent detection of viruses, suggesting that both bacteria and viruses were implicated in ARI. Of different bacterial and viral associations, Moraxella and RSV co-infection was more likely to cause lower respiratory infection with fever. Those who had earliest colonisation with Moraxella or Streptococcus experienced ARI at younger age. Whilst Alloiococcus was most frequent component of healthy NP microbiome, abundance of Moraxella proportionally increased with increasing number of ARI in the first year of life. As regards to the risk of later asthma development, infants who had early Streptococcus colonisation and febrile or HRV-C wheezy, lower respiratory infection by age 12 months were more likely to be atopic at age 2 and chronic wheezers by age 5 years. Interestingly, in this study antibiotic use in preceding four weeks to NP sampling was also associated with abundance of Streptococcus, Moraxella and Haemophilus. This means that early life antibiotic use could determine respiratory microbiota composition for longer periods of time which in itself might be a risk factor for future lower respiratory illness associated with viral infection.

In the cross-sectional GABRIELA study set to assess genetic and environmental factors determining asthma among 6-12 year old children from different rural areas across the Europe, nasal samples of asthmatic children revealed lower microbial richness and greater abundance of genus Moraxella from Proteobacteria phylum compared to controls (31). The association between abundance of Moraxella and presence of asthma was only observed among children who were not living on farms. Furthermore, there was no association between diversity of throat microbiota and asthma presence, suggesting that throat microbiota may be more influenced by immune regulation of lymphoid tissues that can act as a barrier to microorganisms, whilst nasal microbiota seems to be less regulated and more prone to diversity based on environmental exposures.

Moraxella catarrhalis colonisation in children is more common in cooler months and is known to cause biofilm on mucosal surfaces making antibiotics less effective and facilitates co-colonisation with $S$. pneumoniae and $H$. influenzae (30). Colonisation with these three species seems to disrupt nasal microbiota and reduce bacterial diversity (27). Moreover, M. catarrhalis, S. pneumoniae, $H$. influenza as well as P. aeruginosa and $S$. aureus were cultured from adults with asthma both during exacerbations and at periods when symptoms were controlled (28).

Mode of delivery and feeding status affects composition of colonising commensal bacteria within first few months of life which might be critical period for immune system stimulation (27). Different studies have shown that breastfeeding selects for nasal colonisation with predominantly Staphyloccoccus/Dolosigranulum and Corynebacterium whilst formula feeding is associated with abundance of Staphylococccus at 6 to 12 weeks (27). Those infants who have predominant colonisation with Moraxella, Dolosigranulum and Corynebacterium have more stable nasal microbiota during the first 2 years of life. Changes in nasal microbiota are observed during the childhood and over the lifetime with a transition occurring in puberty with Actinobacteria are predominant in adults.

Asthmatic adults have distinctive nasal microbiota with greater abundance of Proteobacteria and Bacteroidetes (32). Moreover, those subjects with more active asthma prone to exacerbations have higher proportion of bacterial genera from Bacteroidetes, Proteobacteria and Actinobacteria phyla, even after adjusting for multiple confounders including allergic rhinitis, recent antibiotic, antihistamine and intranasal corticosteroid use. In numerous other studies presence of Proteobacteria was associated with asthma sever- 
ity and expression of $\mathrm{T}_{\mathrm{H}} 17$ pathway genes (28). The induction of $\mathrm{T}_{\mathrm{H}} 17$ pathway by $H$. parainfluenzae has been proposed as a cause of corticosteroid resistant asthma phenotype and may constitute future therapeutic targets for this asthma phenotype.

Overall, acquisition and maintenance of respiratory microbiome is dynamic process over the lifetime. In particular establishment of respiratory colonisation in the first 2 years of life in combination with respiratory viral infections seems to predispose and later to be associated with current wheeze and asthma. The finding of over-representation of pathobionts such as Moraxella, Streptococcus and Haemophilus in nasal microbiota of children even before they develop asthma is intriguing and this observation needs to be taken further into mechanistic studies to explain possible causality.

\section{Conclusion}

We are beginning to understand how loss of microbial diversity has influenced development of asthma and allergic disease. Rather than being innocent bystanders, gut and respiratory microbiota are crucial determinants of health and disease. Recent advances in scientific methods have enabled us to get insight into complex patterns of colonisations, its constituents and their interactions with host immune system. Discovery of gut-lung axis and lower respiratory microbiota offers an exciting opportunity for further mechanistic studies and potential intervening strategies for childhood asthma prevention. There is a need for well-designed longitudinal studies looking into dynamic aspects of human microbiome acquisition and stability in relation to disease development. What will be more challenging is to find a minimum microbial combination that provokes right immune system programming in early life, which then can be translated into safe intervention for prevention of asthma and allergy. The effect of such possible strategy on established asthma would need to be separately addressed.

Conflict of Interest: The author declares that she has no conflict of interest.

\section{References}

1. Platts-Mills TA. The allergy epidemics: 1870-2010. J Allergy Clin Immunol. 2015;136(1):3-13.

2. Strachan DP. Hay fever, hygiene, and household size. BMJ. 1989;299(6710):1259-60.

3. Jackson DJ, Gern JE, Lemanske RF, Jr. Lessons learned from birth cohort studies conducted in diverse environments. J Allergy Clin Immunol. 2017;139(2):379-86.

4. Yang IV, Lozupone CA, Schwartz DA. The environment, epigenome, and asthma. J Allergy Clin Immunol. 2017;140(1):14-23. Epub 2017/07/05.

5. Schroder NW, Crother TR, Naiki Y, Chen S, Wong $\mathrm{MH}$, Yilmaz A, et al. Innate immune responses during respiratory tract infection with a bacterial pathogen induce allergic airway sensitization. J Allergy Clin Immunol. 2008;122(3):595-602 e5.

6. Ownby DR, Johnson CC, Peterson EL. Exposure to dogs and cats in the first year of life and risk of allergic sensitization at 6 to 7 years of age. JAMA. 2002;288(8):963-72.

7. Fujimura KE, Demoor T, Rauch M, Faruqi AA, Jang S, Johnson CC, et al. House dust exposure mediates gut microbiome Lactobacillus enrichment and airway immune defense against allergens and virus infection. Proc Natl Acad Sci U S A. 2014;111(2):805-10. Epub 2013/12/18.

8. Ege MJ, Mayer M, Normand AC, Genuneit J, Cookson WO, Braun-Fahrlander C, et al. Exposure to environmental microorganisms and childhood asthma. N Engl J Med. 2011;364(8):701-9.

9. Stein MM, Hrusch CL, Gozdz J, Igartua C, Pivniouk V, Murray SE, et al. Innate Immunity and Asthma Risk in Amish and Hutterite Farm Children. N Engl J Med. 2016;375(5):411-21.

10. Prince BT, Mandel MJ, Nadeau K, Singh AM. Gut Microbiome and the Development of Food Allergy and Allergic Disease. Pediatr Clin North Am. 2015;62(6):1479-92.

11. Hoskin-Parr L, Teyhan A, Blocker A, Henderson AJ. Antibiotic exposure in the first two years of life and development of asthma and other allergic 
diseases by 7.5 yr: a dose-dependent relationship. Pediatr Allergy Immunol. 2013;24(8):762-71.

12. Ahmadizar F, Vijverberg SJH, Arets HGM, de Boer A, Turner S, Devereux G, et al. Early life antibiotic use and the risk of asthma and asthma exacerbations in children. Pediatr Allergy Immunol. 2017;28(5):430-7.

13. Semic-Jusufagic A, Belgrave D, Pickles A, Telcian AG, Bakhsoliani E, Sykes A, et al. Assessing the association of early life antibiotic prescription with asthma exacerbations, impaired antiviral immunity, and genetic variants in 17q21: a populationbased birth cohort study. The Lancet Respiratory medicine. 2014;2(8):621-30.

14. Bloomfield SF, Rook GA, Scott EA, Shanahan F, Stanwell-Smith R, Turner P. Time to abandon the hygiene hypothesis: new perspectives on allergic disease, the human microbiome, infectious disease prevention and the role of targeted hygiene. Perspect Public Health 2016;136(4):213-24.

15. Huang YJ, Marsland BJ, Bunyavanich S, O'Mahony L, Leung DY, Muraro A, et al. The microbiome in allergic disease: Current understanding and future opportunities-2017 PRACTALL document of the American Academy of Allergy, Asthma \& Immunology and the European Academy of Allergy and Clinical Immunology. J Allergy Clin Immunol. 2017;139(4):1099-110.

16. Sudo N, Sawamura S, Tanaka K, Aiba Y, Kubo C, Koga Y. The requirement of intestinal bacterial flora for the development of an IgE production system fully susceptible to oral tolerance induction. J Immunol. 1997;159(4):1739-45.

17. Moossavi S, Miliku K, Sepehri S, Khafipour E, Azad MB. The Prebiotic and Probiotic Properties of Human Milk: Implications for Infant Immune Development and Pediatric Asthma. Front Pediatr. 2018;6:197.

18. Arrieta MC, Stiemsma LT, Dimitriu PA, Thorson L, Russell S, Yurist-Doutsch S, et al. Early infancy microbial and metabolic alterations affect risk of childhood asthma. Sci Transl Med. 2015;7(307):307ra152.

19. Zhang B, An J, Shimada T, Liu S, Maeyama K. Oral administration of Enterococcus faecalis FK23 suppresses Th17 cell development and attenuates allergic airway responses in mice. Int $\mathrm{J}$ Mol Med. 2012;30(2):248-54.

20. Chen B, Chen H, Shu X, Yin Y, Li J, Qin J, et al. Presence of Segmented Filamentous Bacteria in Human Children and Its Potential Role in the
Modulation of Human Gut Immunity. Front Microbiol. 2018;9:1403.

21. Bradley CP, Teng F, Felix KM, Sano T, Naskar D, Block KE, et al. Segmented Filamentous Bacteria Provoke Lung Autoimmunity by Inducing GutLung Axis Th17 Cells Expressing Dual TCRs. Cell Host Microbe. 2017;22(5):697-704 e4.

22. Barcik W, Wawrzyniak M, Akdis CA, O'Mahony L. Immune regulation by histamine and histamine-secreting bacteria. Curr Opin Immunol. 2017;48:108-13.

23. Maslowski KM, Mackay CR. Diet, gut microbiota and immune responses. Nat Immunol. 2011;12(1):5-9.

24. Barcik W, Pugin B, Westermann P, Perez NR, Ferstl R, Wawrzyniak M, et al. Histamine-secreting microbes are increased in the gut of adult asthma patients. $\mathrm{J} \mathrm{Al-}$ lergy Clin Immunol. 2016;138(5):1491-4 e7.

25. Klopp A, Vehling L, Becker AB, Subbarao P, Mandhane PJ, Turvey SE, et al. Modes of Infant Feeding and the Risk of Childhood Asthma: A Prospective Birth Cohort Study. J Pediatr. 2017;190:192-9 e2.

26. Guilleminault L, Williams EJ, Scott HA, Berthon BS, Jensen M, Wood LG. Diet and Asthma: Is It Time to Adapt Our Message? Nutrients. 2017;9(11). Epub 2017/11/09.

27. Bomar L, Brugger SD, Lemon KP. Bacterial microbiota of the nasal passages across the span of human life. Curr Opin Microbiol. 2018;41:8-14.

28. Chung KF. Potential Role of the Lung Microbiome in Shaping Asthma Phenotypes. Ann Am Thorac Soc. 2017;14(Supplement 5):S326-S31.

29. Bisgaard H, Hermansen MN, Buchvald F, Loland L, Halkjaer LB, Bonnelykke K, et al. Childhood asthma after bacterial colonization of the airway in neonates. N Engl J Med. 2007;357(15):1487-95.

30. Teo SM, Mok D, Pham K, Kusel M, Serralha M, Troy N, et al. The infant nasopharyngeal microbiome impacts severity of lower respiratory infection and risk of asthma development. Cell Host Microbe. 2015;17(5):704-15.

31. Depner M, Ege MJ, Cox MJ, Dwyer S, Walker AW, Birzele LT, et al. Bacterial microbiota of the upper respiratory tract and childhood asthma. J Allergy Clin Immunol.. 2017;139(3):826-34 e13.

32. Fazlollahi M, Lee TD, Andrade J, Oguntuyo K, Chun Y, Grishina G, et al. The nasal microbiome in asthma. J Allergy Clin Immunol. 2018;142(3):83443 e2. 